\title{
Efficient Node Monitoring Mechanism in WSN using Contikimac Protocol
}

\author{
Shahzad Ashraf, Mingsheng Gao* \\ College of Internet of Things Engineering \\ Hohai University Changzhou Campus \\ Changzhou, Jiangsu, P.R China
}

\author{
Zhengming Chen \\ College of Internet of Things Engineering \\ Changzhou key Lab. of Orthopedic Implants Digital \\ Technology, Hohai University Changzhou Campus \\ Changzhou, Jiangsu, P.R China
}

\author{
Syed Kamran Haider \\ College of Internet of Things Engineering \\ Hohai University Changzhou Campus \\ Changzhou, Jiangsu, P.R China
}

Zeeshan Raza

Department of Electrical Engineering

University of Lahore, Lahore Pakistan

\begin{abstract}
Wireless Sensor Network is monitored with ContikiMAC Cooja flavor to diagnose the energy utilization ratio by nodes and the fault detection process in distributed approach; adopted the Low power Listening (LPL) mechanism with ContikiMAC to prolong the network's lifetime. LPL locate the root cause of communication issue, get rid of the interruption problems, and get back normal communication state. The LPL mechanism reduces the energy utilization in centralized and distribute approaches. Even more, the distributed approach is best suited for network monitoring when energy utilization is main objective in the presence of LPL. It is also important how soon the faulty node can be detected. In this case, latency has vital contributions in monitoring mechanism and latency is achieved by developing the efficient faulty node detection methodology.
\end{abstract}

Keywords-Wireless sensor networks; low power listening; ContikiMAC; Cooja

\section{INTRODUCTION}

At large scale the human centered appliances are advanced by the remote sensing technology. In-order to provide the accurate judgment, at specific places where human presence is not possible WSN provides the diversity in communication. In Various situations, \& scenarios the WSN are deployed whether for indoor \& outdoor usage, low and high span of environmental vigilance, the health monitoring.

Usually indoor appliances are deployed on small area. Target is to measure the sensing variables and transmit to the sink, a device received the data packets provided by nodes from different locations and sink act as a gateways for providing the access to the Internet and might add some functionalities such as data caching [1]. WSN always have a variety of limited resources like communication bandwidth, memory, reckoning capability \& energy etc. The major task is to provide the communication link which is said to be as the "Not a every bodys' cup of tea". Due to environmental effect and the quality factor of the sensors it is essential to maintain the sensor network efficiently to work properly. Nodes and links' status is required to be probed regularly and the critical faults should be detected within a bounded time period to provide appropriate countermeasures and prevent unexpected consequences due to network dysfunctions [2]. For this purpose various type of node information e.g. residual energy and packet transmission succession rate, are required to evaluate the network health. In-order to keep a close eye on network performance, the network frequency probing and the parameter monitoring is the important point. By utilizing existing information leads to the fault occurrences without generating extra packets is also crucial in monitoring environment.

The work is going on to design the intelligent network routing protocol for WSN. Routing provides the best suited pathway to carry the data and much expertise are required to accrue the best routing phenomenon that prolongs the network life. WSN is categorized according to its applications and requirements; hence protocol choice depends on demand of the application. Medium Access Control (MAC) protocol has great role in data routing, as it maintain the structure of routing table that grant access to different sensor nodes to use the channel and medium [3]. The choice of exact and proper MAC protocol is not easy in some cases because every application have different scenarios and models. MAC is responsible to render the best medium capacity and get rid of collision. In real world deployment, energy efficient MAC protocols lead to a great difference in forms of nodes power utilization. Energy efficient MAC protocols were introduced to WSNs area with the aim to decrease the waste of node's energy in idle listening state which increase the network's lifetime and this mechanism is known as sleep wake system [4]. In some books it is also referred as Duty Cycling mechanisms and commonly classified as synchronous and asynchronous schemes.

Several MAC duty cycle protocols have been proposed during the last decade to address specific WSNs requirements and constraints such as a low energy consumption linked to battery operated nodes one of them is a Radio Duty Cycle (RDC) MAC protocol which try to reduce the energy consumption by allowing a node to keep its radio-transceiver

\footnotetext{
*Corresponding Author
} 
off most of the time [5]. This allow a node to avoid to keep the radio on unnecessarily i.e. when not involved in any transmission Idle listening is used to solve such problem in which RDC MAC forces node to switch its transceiver between short active(listen) periods and long inactive (sleep) periods. Similar to it around the neighbors, packet transmission is listened by using the periodical wake ups in ContikiMAC protocols as it behaves as radio duty cycling protocol. It works in such a way that If at the time of wake up interval, received the packet transmission, the receiver remain in active state and if the packet is received it send the acknowledgement [6].

In order to send the packet, sender sends packet time after time till it receives the acknowledgment by the receiver that sent a broadcast, is not the form of output in link layer ACK. Rather, sender sends packet, time after time all along full active wake up period, that every neighbour accepted it [7]. Fig. 1 below shows the connection mechanism of ContikiMAC protocol.
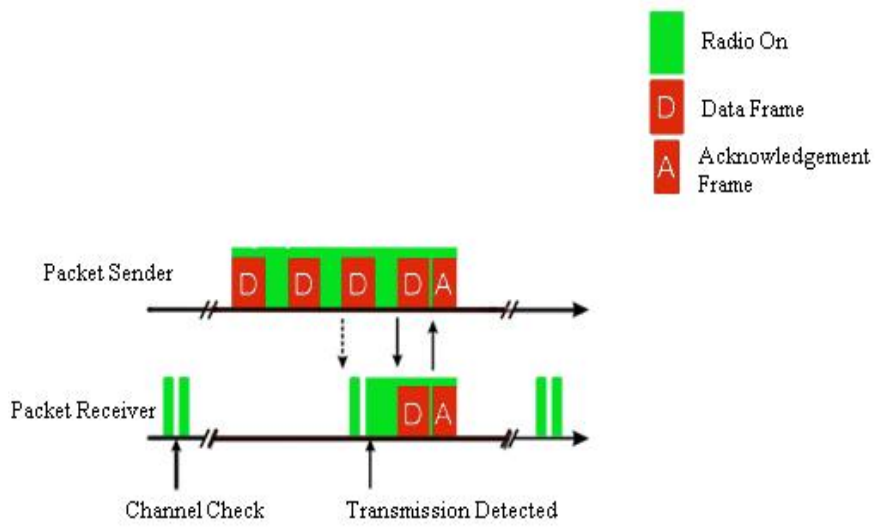

Fig. 1. ContikiMAC operation mechanism.

The power efficacious wake up system of ContikiMAC demands the smooth timing among the transmission. The node's sleep-wake mechanism is carried out by the two well know techniques the Low Power Listening (LPL) and Low Power Probing (LPP) used for asynchronous process. To understand the WSN monitoring mechanism, consider the bunch of various activities launched by the sensor nodes to look after the actions of their next door friends' node, known as monitored nodes [8]. The target is to just odd man out the unusual activities in network regarding the nodes' behavior, as if there is any non-cooperative node; also, the availability of battery timing, sensing board issue, adjustability of sensor nodes and the wireless communication link quality including high collision rates, jamming attacks, etc. All these challenges are based on WSNs' characteristics. The monitoring targets are the intended and non-harmonious node.

To propose the WSN monitoring mechanism a framework is suggested to pattern the MAC protocol. The idea is rest on a heartbeat mechanism to detect node failures in both centralized and distributed scheme. The LPL is used to represent energy efficient MAC protocols instead of the higher overhead, difficult to implement synchronous methods. They took the help from ContikiMAC protocol based on the LPL mechanism. The suggested mechanism focuses on energy utilization $\&$ the detection factors.

The remaining sections are arranged as, Section II possessed the related work, and implementation is given in Section III, while Section IV is highlighted with performance judgment. Finally, Sections V and VI possessed the conclusion and future work, respectively.

\section{RELATED WORK}

Node behaves, as the self-organizing pillars of the WSNs. The task of monitoring is accomplished by the mutual cooperation of the nodes in WSN. Layered clustering of network structure is used when the size of network is broader [8]. Basically network fault monitoring system of WSN is composed on four layers as given below:
1. Application development layer
2. Data manager layer
3. Network layer
4. Fundamental layer

Starting from bottom the foundation layer is considered as core of the sensor node, containing the hardware \& software of every sensor like perception gear, memory \& embedded processor, communication gears, embedded O.S and specially designed database architecture. This layer is responsible of detecting sensing nodes, capturing of objects data and send it to relevant instance. The ${ }^{2}$ nd network layer, the core layer of the network, conceive sensor node and data capture technique, provides communication among diversity of sensing broader collaborative tasks. It supports the hand shaking mechanism in the network \& provides the pace for resources like hardware $\&$ software. Core of the data management and processing of sensor is performed by the ${ }^{3}$ rd layer as Data Manager Layer. Keep records of sensory data; manage the storage structure, retrieval of data, analysis and mining, query support. The last top and upper most layers provides the support for software development tools as its name depict the application development layer so it supports to rest of the layer to provide the development environment for different applications of WSN [9].

In classical, network monitoring system is divided into two approaches, first one is the centralized, where sink node of the WSN receives the node information of all aspects, the centralized decision making system closely decided whether fault is reported or not, hence this approach determine the root-cause of faults using a decision tree. Although it is much better mechanism but it has some drawbacks like in the case of getting heavy amount of data. Second approach is distributed, where sink do not receive information from all nodes but nodes by themselves have responsibility to monitor the system in order to achieve more energy efficiency [10].

It is a major hardline for WSN to make sure the smooth \& low tariff communication link between node to node with balanced data delivery. The uneven load distribution disturb the network lifetime across the network. Nodes located near to the sink suffer with high energy deficiency rather than the nodes away from the sink. Network may goes down for undefined period due to the unavailability of the communication link with sink. The network demands the

\footnotetext{
*Corresponding Author
} 
unbroken connectivity and small delay may be accepted. For long periods the nodes can't be sleep time to time and they must be alive for unaltered functionality. All this can be achieved by the unique MAC protocols that save the nodes energy and fixed the workload for each node in the network. One of the best approaches to reduce the node energy consumption by scheduling the node's radio state on and off is the Duty Cycling mechanism [11].

The goal is to make nodes operate in low duty cycles, spending most of their time sleeping and waking up only when engaging in packet transmissions, to diminish energy waste in idle listening and overhearing conditions. Up to $50 \%$ unnecessary power consumption can be reduce by turning off the nodes' radio, during the off duty interval of the nodes. There are two approaches of MAC duty cycling mechanism, the synchronous \& asynchronous. The sleep and wake up time schedule of the nodes are created by the Synchronous approach while transmitter initiated \& receiver initiated are the further division of the Asynchronous approach [12]. The regular transmission packets with short preamble data packets themselves are sent by the node in transmitter initiated approach, in expectation any of them collide with listening time of the destination node. While frequent packets requests are sent by the node in receiver initiated approach with acknowledgment to notify the neighboring nodes regarding expedition of node to gain the packets. The complexity of global time synchronization can be reduced by employing the schemes which nodes only agree on a local common clock within neighbors and use asynchronous methods as intercluster communication [13]. In asynchronous approach, among nodes, the communication is carried out due to Low Power Listening Protocol (LPL), to such a degree vanish the overhead for time synchronization. In designing of energy efficient MAC protocol, the attributes of the LPL are greatly explored because LPL possessed the common mechanism. Time to time node in LPL look for receive checks and if it do not detect any channel activity it put off its radio. During channel busy period, node remains in active state to accept the packets. This technique is highly susceptible to false wakeups, especially in noisy environment, Noise may causes to triggered the false wakeup and node do not receive any incoming packet but due to the false triggered it may receive unusual duplicate unreal packets [14].

The ContikiMAC protocol improves sleep wake mechanisms, nodes used by ContikiMAC, periodically wake up to sense radio activity on the channel using Clear Channel Assessment (CCA) mechanism. Unicast transmission is done by having the sender keep transmitting the same packet until it receives an ACK from the intended receiver. The maximum time of repeating transmission is the full wake-up interval which has been proven that the packet reception is guaranteed if the sender sends packets throughout this interval. In the case of broadcast transmission, there is no ACK mechanism, thus the sender must send packets continuously for the full wakeup interval [15]. The ContikiMAC fast sleep optimization scenario is given in Fig. 2. Before the time ti, if silence period do not detected, receiving node turn towards sleep mode, and if such silence interval is longer than ti, then receiving node remain to a sound sleep, the receiving node travel back to sleep mode if no packet is received after the silence interval, though radio activity is alive [16].

According to the author in [17], the energy level is checked by each node itself and forwards the message to central object if the state change is occurred. On the other side the author [18] developed a tool to detect the fault. The scheme Based on sink that utilized the message spreading technique to choose the event related data $\&$ present states of the network.

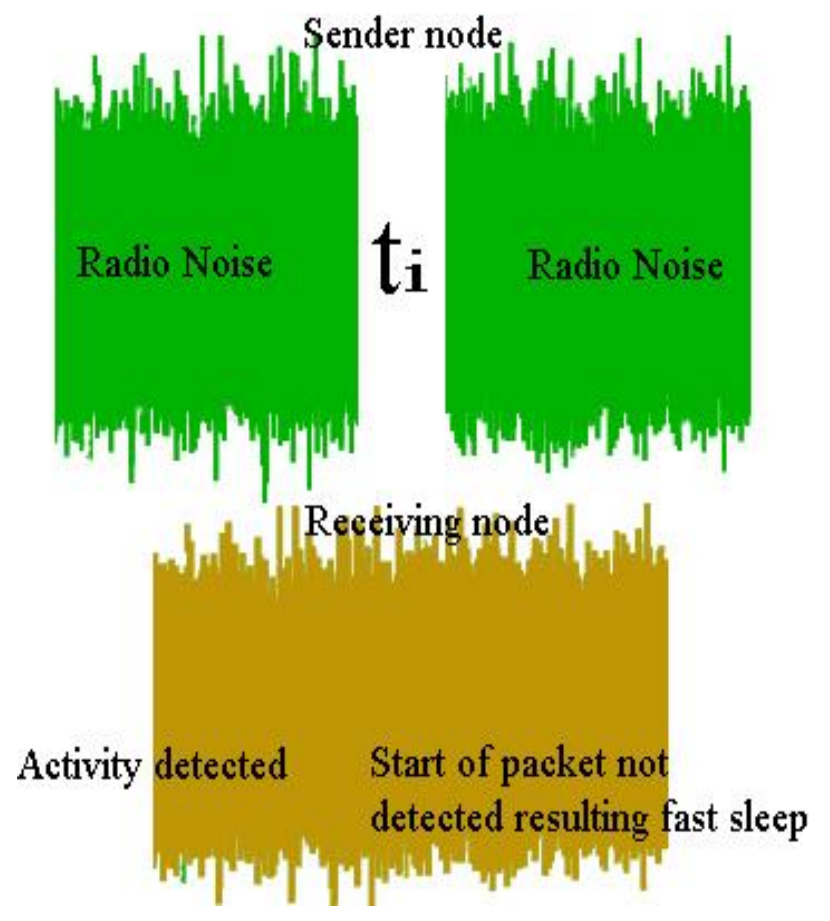

Fig. 2. ContikiMAC's duty cycling process.

According to the distributed approach, it is decided locally to detect the fault, for that only few messages are enough to transmit towards central object. While another author in [19] worked on two phase self-monitoring mechanism (TP) which highlights the node self-health monitoring system by this individual node can monitor its own health and the neighbors node health too, upon the state report it explore the local fault detection mechanism.

\section{IMPLEMENTATION}

Taking the help from Contiki OS, possessed the LPL based medium access control mechanism. In-order to monitor the network performance proceeds to judge the results of network monitoring with and without LPL implementation. Centralized and distributed approaches are considered to check the impact of LPL on the performance of the network. The system is composed of heartbeat messages mechanism which is a general and well known network monitoring approach, as node periodically sends packets to its monitoring node which could be the powerful sink or any node in the network. Every node sends the heartbeat messages to the sink after every second in the centralized monitoring scheme. Packet collision is avoided by adding the small amount of delay time before transmitting the message. The process is explained in below Fig. 3.

\footnotetext{
*Corresponding Author
} 


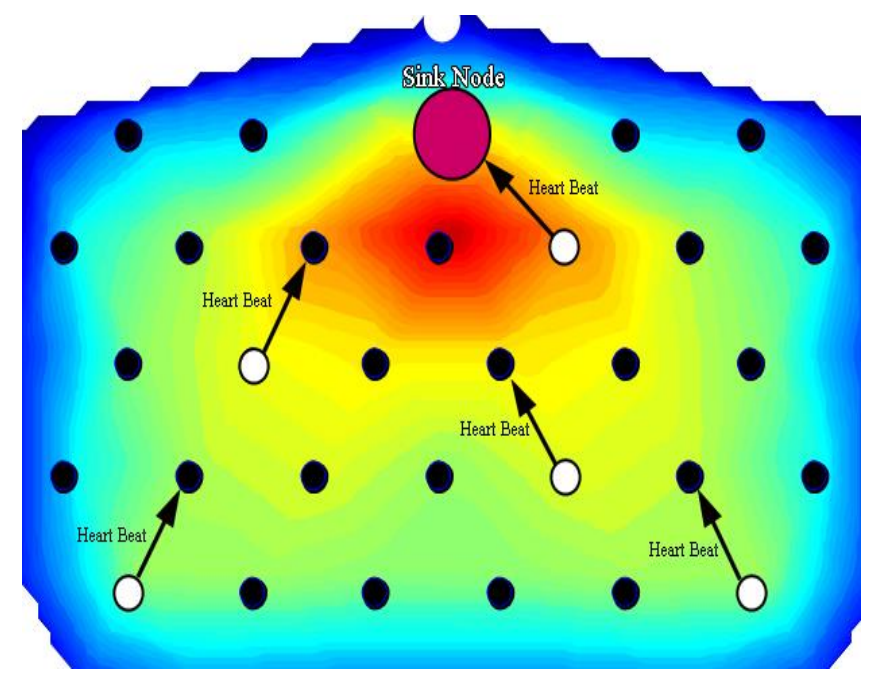

Fig. 3. Heartbeat message mechanism in centralized approach.

As sink receive the heartbeat signal \& send the acknowledgment signal to the sending node, resulting the sending node shall be referred as active node and its monitoring session shall be started. If the time difference between nodes current time and nodes recent timestamp is greater than the monitoring interval then node shall be considered as in idle state. Hence sink shall monitor all nodes and share the status with monitoring administrator.

While in distributed approach the case is change, any node can take the responsibility of monitoring, not the all node. Every node just broadcast to its neighboring one. It is just like a tree fashion the credentials of monitoring node is embedded in the message by which the child node to be known. When a monitoring node starts monitoring a new node, it will transmit a message to inform the sink. The sink assumes that all nodes are currently active unless there is a report of node's inactive status as illustrated in below Fig. 4. While rest of the process is as it is like in centralized approach.

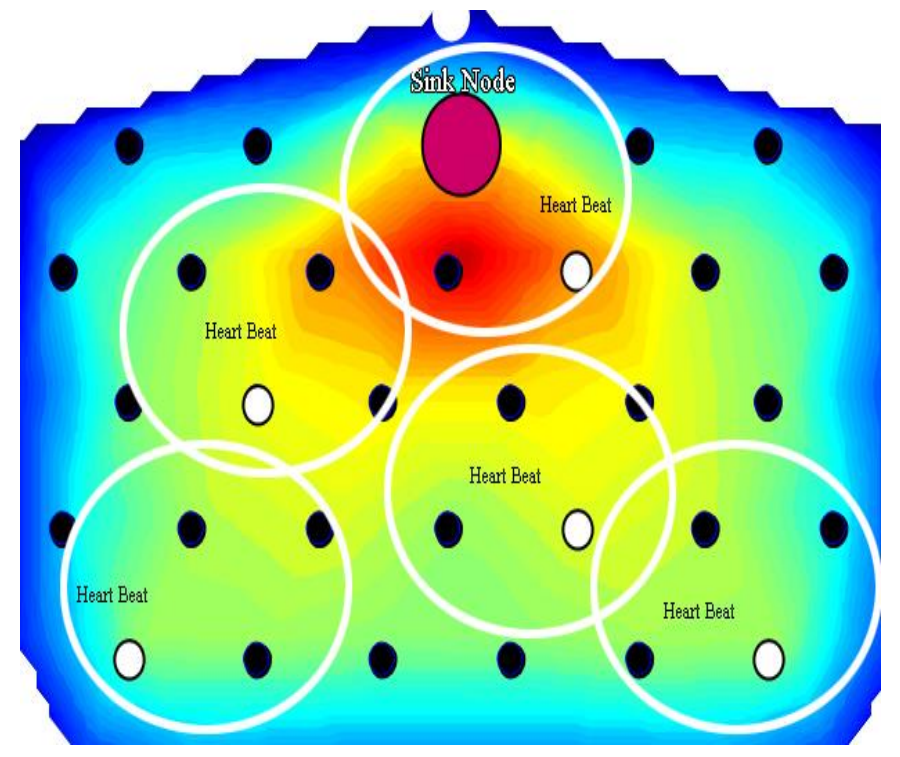

Fig. 4. Heartbeat message mechanism in distributed approach.
Considered the parent node as sink or monitoring node, the whole phenomenon is managed by routing algorithm but if the parent is going to be change the previous monitoring node will automatically stop the monitoring process after receiving a heartbeat containing a different node address from its child node in order to prevent false alarms.

\section{Performance Judgment}

The network performance has been evaluated on Contiki's Cooja simulation. Contiki is a Linux, Ubuntu based O.S for the Internet of Things.

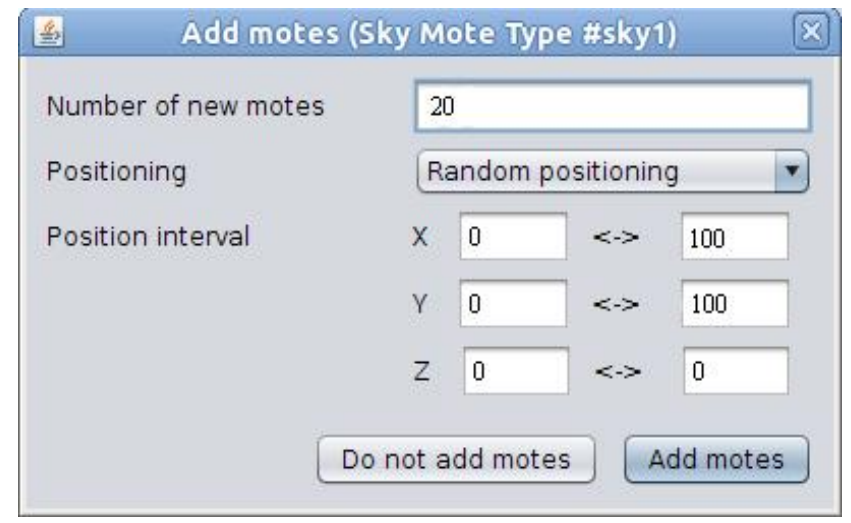

Fig. 5. Addition of number of nodes in Contiki Cooja.

It works as virtual machine that is bound with VMWare player, whereas Cooja is a Contiki Java based discrete event simulator. The sensor nodes are represented by the Motes. It is used to inspect the network behavior, very fast and support the longer networks.

The simulation began by entering the parameters values. Added the 20 number of nodes including the sink in the form of motes in Cooja is shown in Fig. 5 above. The random positioning has been chosen whereas position intervals are set as 100 for $\mathrm{x}$ and $\mathrm{y}$ while $\mathrm{z}$ coordinate is 0 . While in Fig. 6 the number of nodes are flowed out in random position.

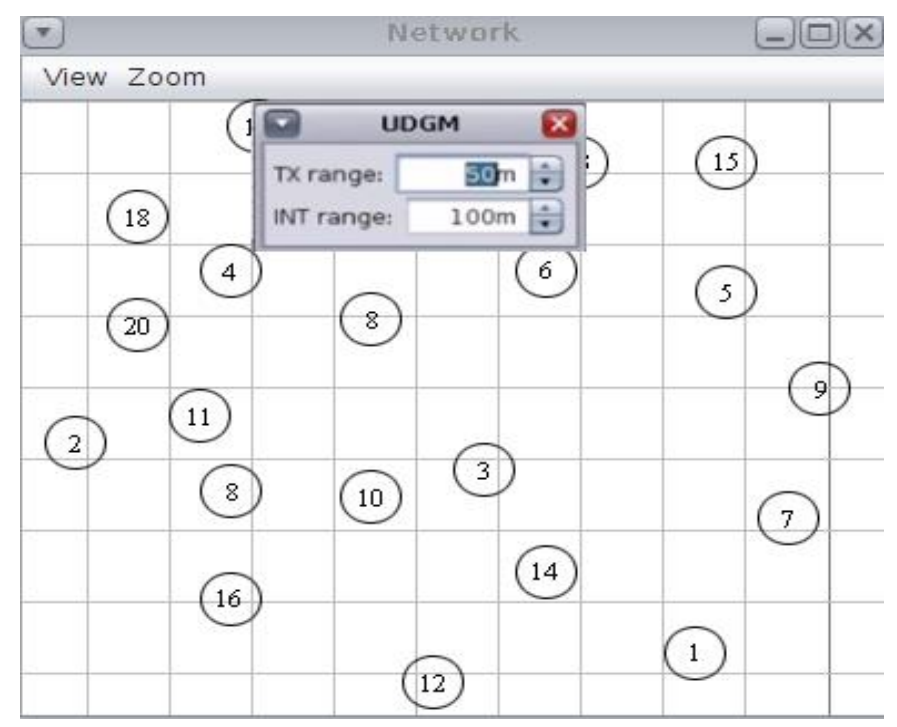

Fig. 6. Nodes distribution in random fashion.

\footnotetext{
*Corresponding Author
} 


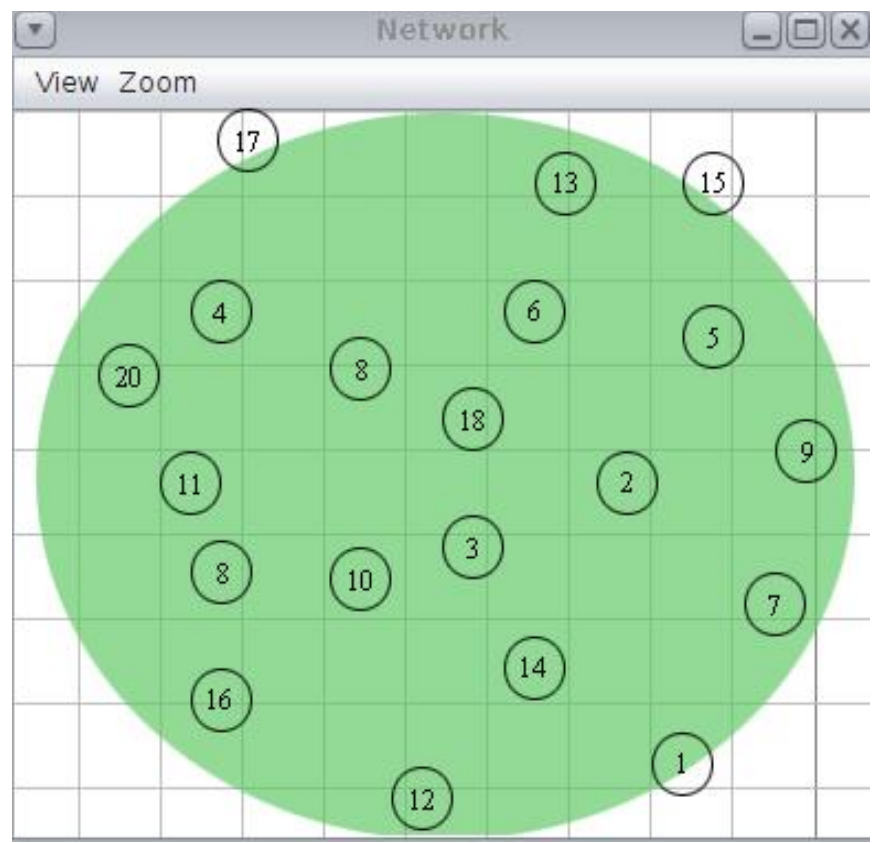

Fig. 7. Nodes in radio range.

Transmission range is entered as 50 meter while Interference is 100 meter. More the Heartbeat time is fixed at 10 second while monitoring time range is 35 seconds. It is to be make sure the all the nodes should be in radio range and it can be verified as by clicking on view menu and radio Environment and just click on any node it will show the range boundaries in green color or just change the Transmission Range (TX) and Interference Range to adjust the nodes, illustrated in Fig. 7.

Now by starting the simulation it will begin to broadcast the messages among the nodes as shown in Fig. 8 (a)-(c).

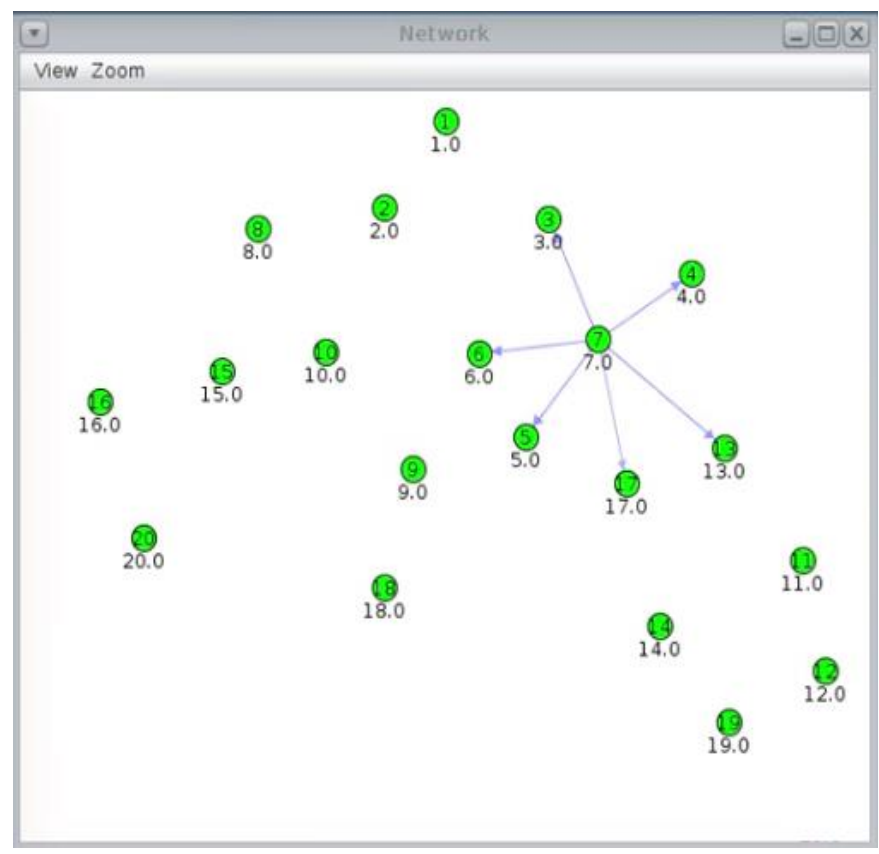

(a)
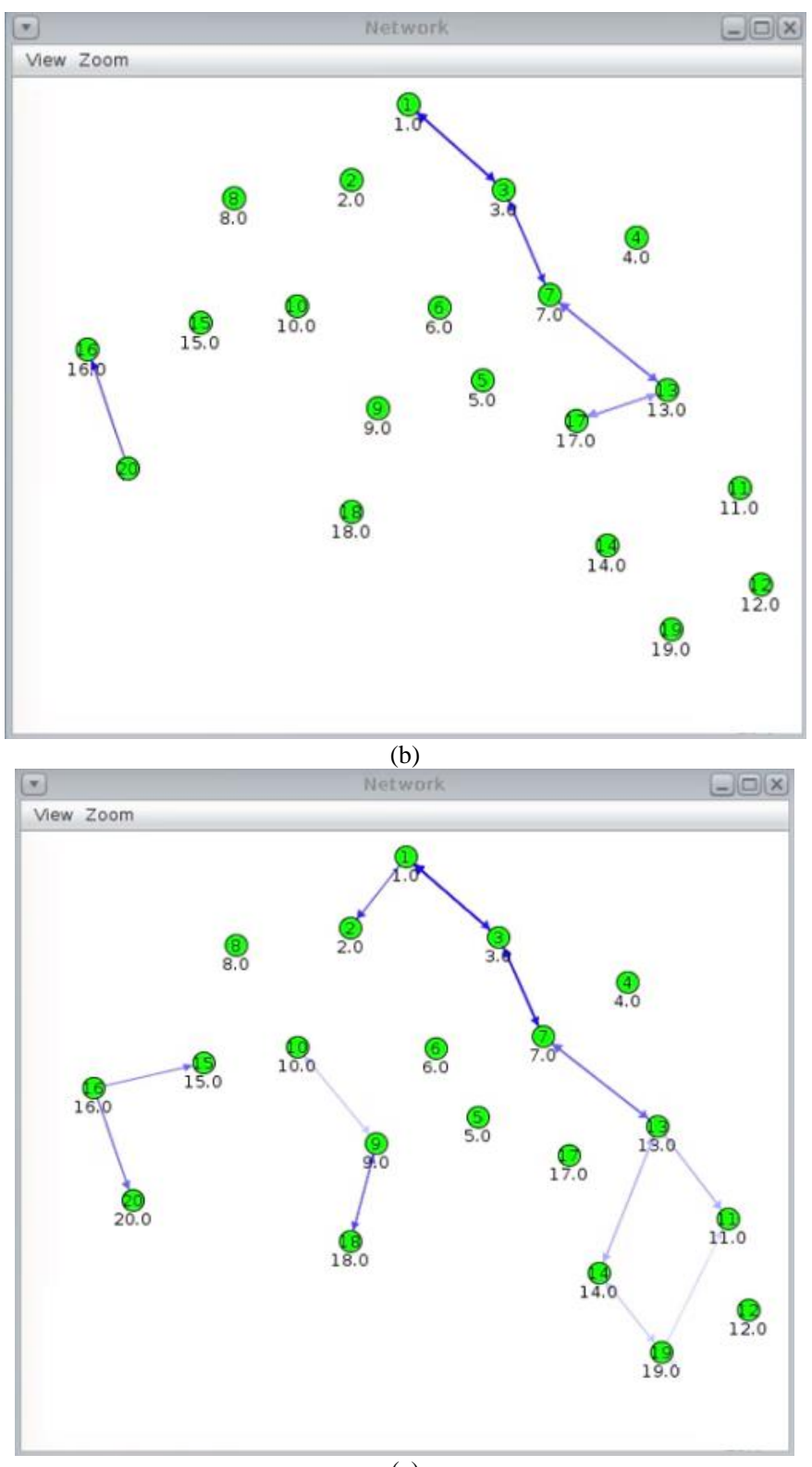

(c)

Fig. 8. (a)-(c) Node begun to broadcast message.

Every node is following its path towards sink. Each node chose the nearest node with the smallest number of hops to the sink as its parent node. If there is fault in any node, the rerouting process shall be activated [20]. The comparison of two different results are carried out between ContikiMAC, having LPL mechanism \& IEEE802.15.4 or VoidMAC in the absence of LPL.

The point of monitoring in simulation is to monitor the average power consumption of each node and the detection of fault accuracy. The monitoring observation is focused on fault detection \& the generation of unreal alarm ratio. If the greater number of fault detection reported it means that overall monitoring process is working fine. In addition the unreal false detection ratio should be lower or minimized as it just indicates the sensitivity of the network $\&$ the unreal status of

\footnotetext{
*Corresponding Author
} 
the nodes. Secondly the lesser power usage is also highlighted because it will prolong the network life time.

\section{A. Energy Utilization}

It is calculated by taking the ratio of CPU beats in every state of sensor node among the fixed given interval. This job is done by the Energest module of contiki. The sensor nodes consist of Transmission (TX), Receiving (RX), CPU Idle and Sleep or Lower Power (LPM) mode. The states of the node are monitored by these parameters. It is observed that in LPM mode the node utilize less amount of energy as compare to other, so overall energy utilization is shown in Table 1, from where it is certified that nodes in LPM state is much better than the nodes in CPU idle state due to the less consumption of energy.

TABLE I. NODE ENERGy UTILIZATION STATES

\begin{tabular}{|l|l|}
\hline Node states & Energy Utilization \\
\hline Transmission (Radio TX) & $17.7 \mathrm{~mA}$ \\
\hline Receiving (Radio RX) & $20 \mathrm{~mA}$ \\
\hline CPU Idle & $1.8 \mathrm{~mA}$ \\
\hline Low Power Mode (LPM) & $0.0545 \mathrm{~mA}$ \\
\hline
\end{tabular}

Fig. 9 shows the energy consumption by the nodes. It is the depiction of network monitoring in the presence of ContikiMAC and VoideMAC, it shows evidently that having LPL process it works fine and but during idle state node losses some energy so there is a slight difference in power consumption if avoided the LPL in both centralized and distributed approach.

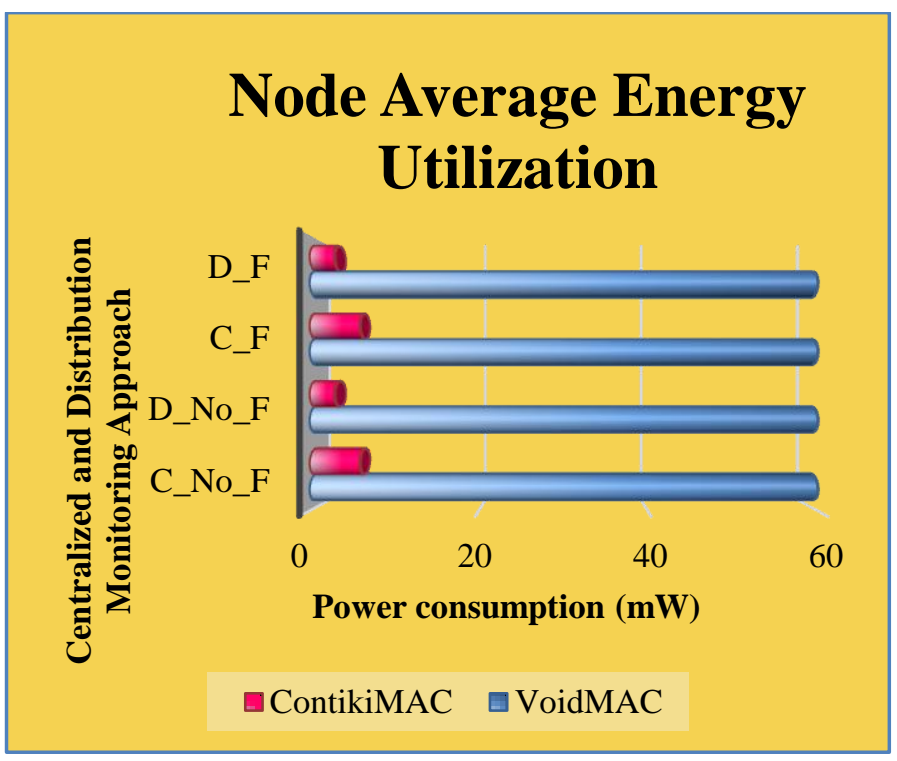

Fig. 9. Power consumption by the nodes in both approaches.

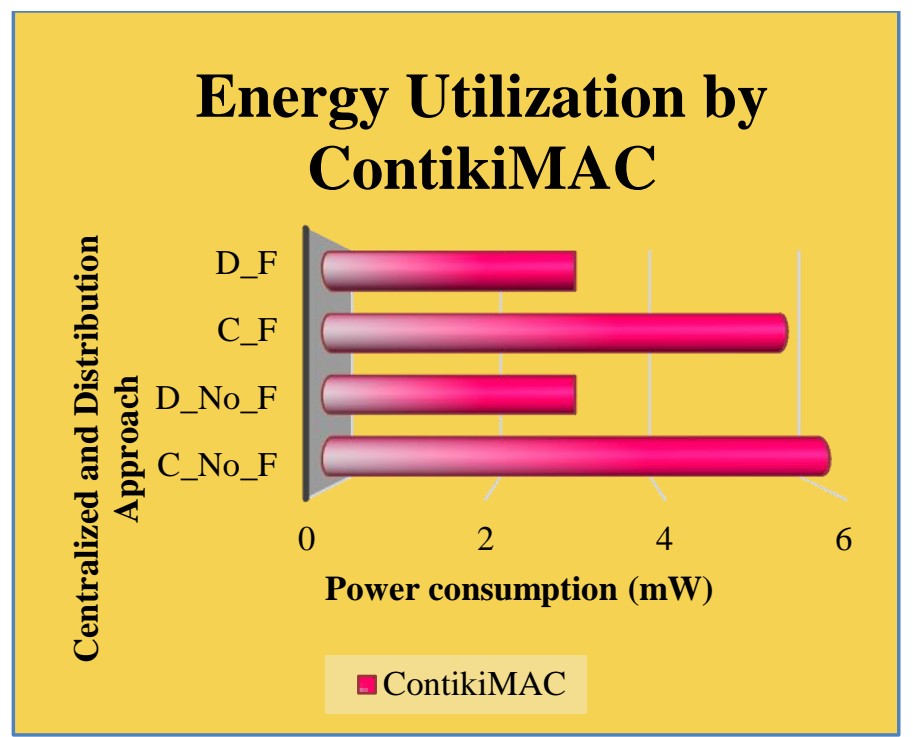

Fig. 10. Power consumption by ContikiMAC.

In contrast to centralized approach, in distribute approach the ContikiMAC utilize only half of the power, thus this approach is more suited than the centralized as appears in Fig. 10 above.

ContikiMAC proves the principle of low-power listening but with better power efficiency. The lower the duty cycle, the longer the nodes batteries, as the most important contribution to the ContikiMAC's reduction in duty cycle is the fast sleep optimization at the heart of the wake up procedure. The negligible energy is consumed when the node sleeps while most energy is consumed during listening (LISTEN), transmitting (TX) or receiving (RX) states.

\section{B. Determination of Adhesion}

It is the process of accurate network activity detection and requires some network related credentials like current State of the Network, denoted by SN, the real Time of the detection represented by $t$, now if time beat is 1 for node $n$ in the network it means the node is alive and possessed the passage up to sink. The status of the sink is denoted by $R_{n}, t$, if the $R_{n}, t$ is 1 it automatically indicates that node is active in current time hence it may be dead in 0 time.

In-order to detect the fault accurately, the idea of fault detection and false alarm ratio is proposed. Fault detection is analyzed as the ratio of the time duration where fault is identified in the mean time of the total fault appears. The detection of fault appears, if the value of $\mathrm{SN}_{n}, \mathrm{t}$ and $\mathrm{Rn}, \mathrm{t}$ are to be 0 , whereas the false alarm ratio can be described as the ratio of the total time having dead node reported and false alarm is obtained when $\mathrm{SN}_{\mathrm{n}}$, t becomes 1 whereas $\mathrm{Rn}, \mathrm{t}$ remain equal to 0 . Fig. 11 shows the absolute scenario.

\footnotetext{
*Corresponding Author
} 


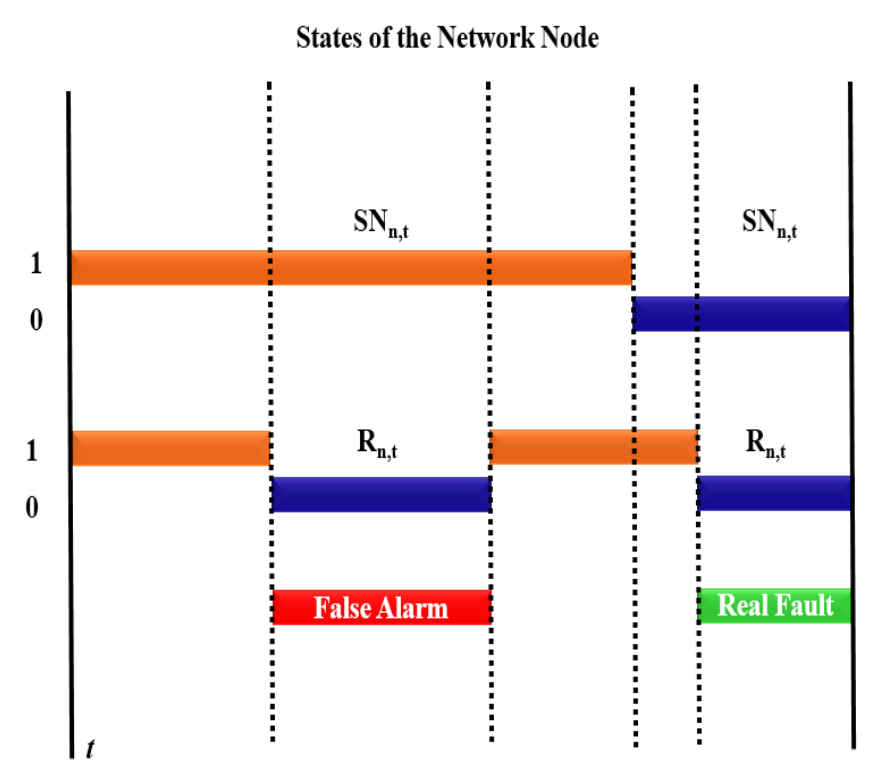

Fig. 11. Generation of real Fault detection and false alarm ratio.

It is important to see how sooner, the fault in the node can be detected by the network, is a major challenge and it can be resolve by determine the latency in the network. For this the Direction Algorithm is developed to determine the faulty node in network. The message packet is broadcasted around the neighbor node by the sink node to odd out the faulty node. The receiving nodes send the ACK packet to the sink. Consider parameters

$$
\mathrm{V}=\{\mathrm{v} 1, \mathrm{v} 2, \mathrm{v} 3, \ldots \ldots \ldots . \mathrm{vn}\}
$$

$\mathrm{V}$ represent the number of nodes while $\mathrm{S}$ is the Sink node. The contents of broadcast message are given in Table 2 below. Each message possessed the Probelist with all node related information, stored in a table, its initial value is set as $\alpha$, as sender node received the acknowledgment by the receiving node, updates their table regarding L-node value and decide whether to decision by itself regarding further forwarding of data packet or not. The process continues until it finds the dead node, as the acknowledgment packet will not receive in the predefined time $\tau$ and the broadcast message shall be paused. Overlapping is reduced by the random decision among the Probepath.

The defected nodes are exposed by attending the series of Algorithm 1.

TABLE II. BROADCAST MESSAGE INFORMATION

\begin{tabular}{|l|l|}
\hline Message Packet & Description \\
\hline B-Id & Broadcast packet Identification \\
\hline S-id & Sender node Identification \\
\hline R-id & Receiving node identification \\
\hline L-Node & Live node available \\
\hline T-Interval & Real time to broadcast the message \\
\hline
\end{tabular}

\section{Algorithm 1: Detection Process of Faulty Node}

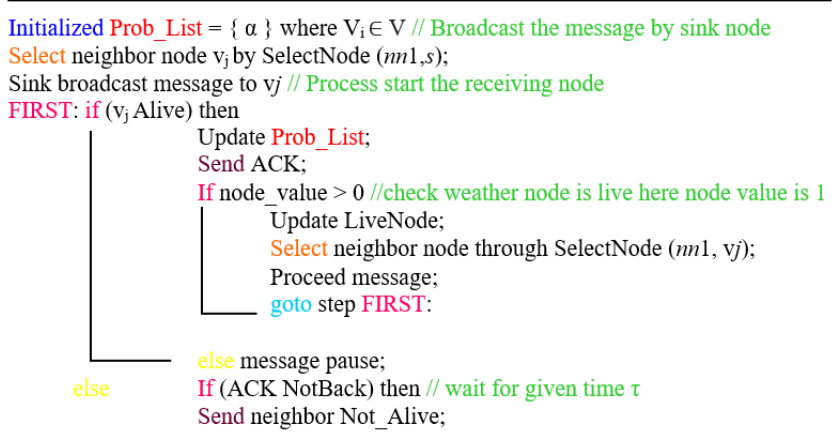

In Fig. 12, it is shown that how fast the latency works against different number of nodes available in a network. It can be observe that latency increases as number of node increases but at some point due to environmental factors it slow down and again get adjusted. While in Fig. 13, the probability of faulty node is illustrated and it is shown that how fast the latency detects targeted faulty nodes. The Latency shall increase if failure probability ratio is lower, resulting lesser chance to detect the faulty node path. Aftermath greater latency is observed against lower probability of sick nodes.

More, by adopting the VoidMAC it shows the false alarm ratio approaches to zero as depicted in Fig. 14.

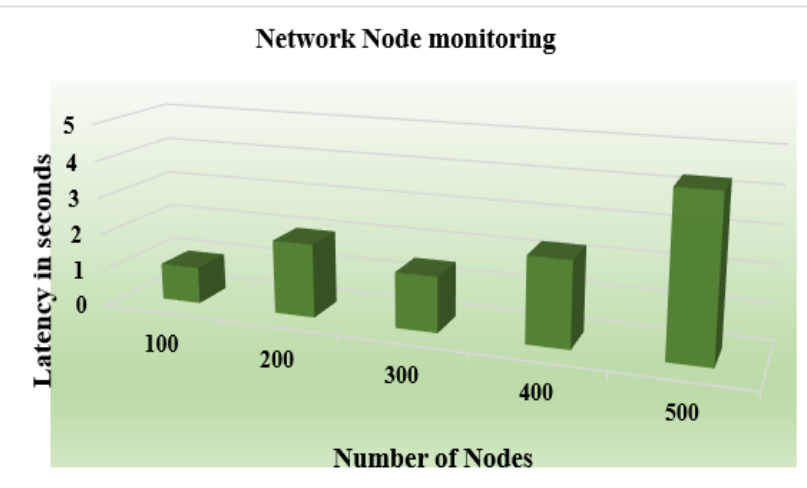

Fig. 12. Latency observation in monitoring session.

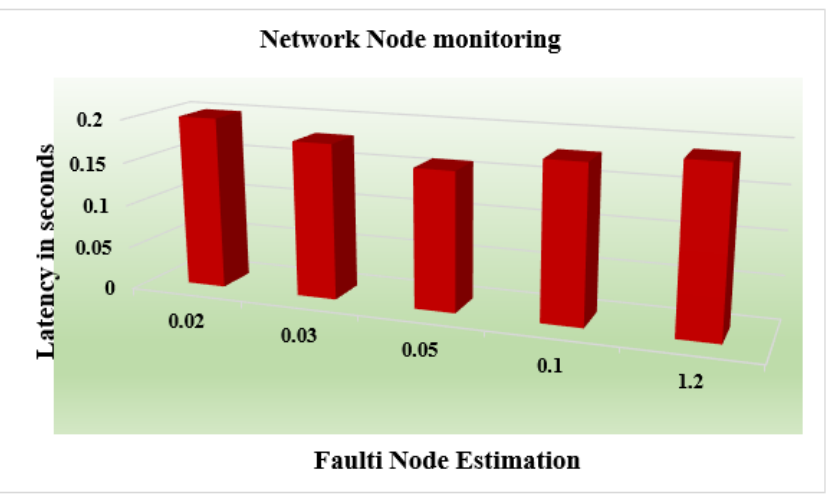

Fig. 13. Latency observation in monitoring session.

\footnotetext{
*Corresponding Author
} 


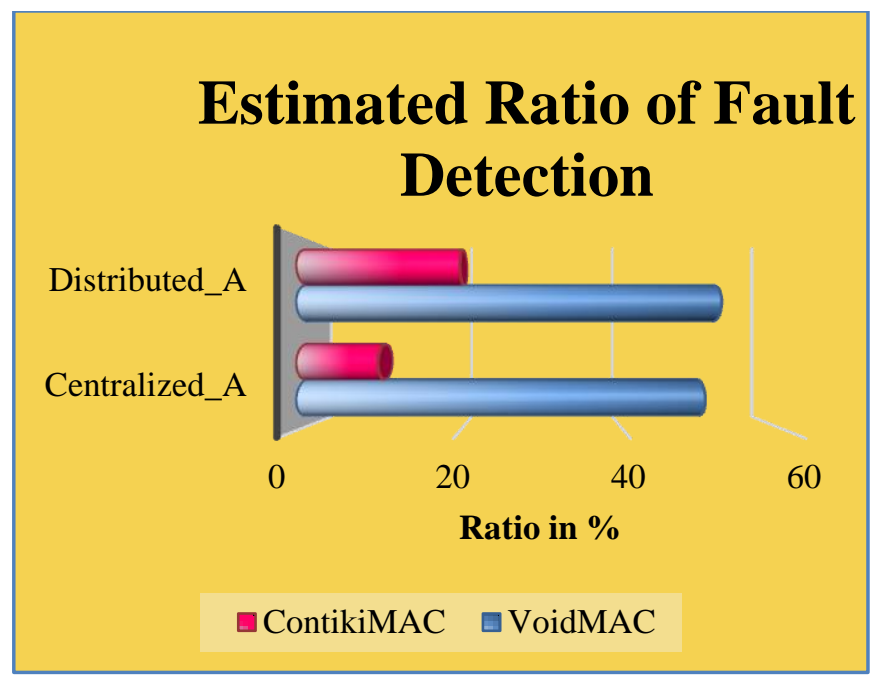

Fig. 14. Latency observation in network monitoring session.

From all results it is observed that by adopting the LPL in centralized and distributed approaches node utilized the much lesser energy, as in LPL sleep mode do not utilized higher energy as compare to idle state. Lower power consumption rate of the network monitoring process will cause less impact on WSNs applications and prolong the lifetime of the network. On the other hand there are some drawbacks of LPL as it generates much duplicate packets for faulty node probing. The large number of packet transmissions generated by the LPL mechanism denotes higher chances of packet collisions, resulting in more losses of packets.

\section{CONCLUSION}

The node faults are estimated by Probe based algorithm, the node make decision by own about the failure state. The Probepath is associated with sink and node failure information is disseminated towards the neighboring nodes. It is observed that the average power consumption of the network by adopting LPL mechanism is tremendously lower, thus it prolong the network's lifetime. Distributed approach is best suited with LPL for best performance in terms of low power utilization.

\section{FUTURE WORK}

It is suggested to develop efficient node detection method which should warn the node failure time before its life end. It is also suggested to investigate the causes of node failure in real time scenario. Duplicate packet transmission consumes much energy, urge to develop the intelligent packet transmission methodology to avoid such situation.

\section{ACKNOWLEDGMENT}

The whole work is partly supported by the National Natural Science Foundation of China under Grans 61772172 and 61671202 and Changzhou City Key Laboratory of Orthopedic Implants Digital Technology.

\section{REFERENCES}

[1] Fayçal Ait Aoudia, Matthieu Gautier, Michele Magno, "A Generic Framework for Modeling MAC Protocols in Wireless Sensor Networks", (2017), IEEE/ACM transactions on networking, VOL. 25.

[2] Sijun Dou, Danpu Liu, "A Reliable MAC Protocol for Hybrid Wireless Sensor Networks", (2016), IEEE International Conference on Internet of Things (iThings).

[3] Fayez Alfayez, Mohammad Hammoudeh, Abdelrahman Abuarqoub, "A survey on MAC protocols for duty-cycled wireless sensor networks",(2015), AWICT.

[4] Thanh Dinh, Younghan Kim, Tao Gu, Athanasios V. Vasilakos, “An Adaptive Low-Power Listening Protocol for Wireless Sensor Networks in Noisy Environments", IEEE systems journal.

[5] Mathieu Michel and Bruno Quoitin, "Technical Report : ContikiMAC performance analysis", (2016), rXiv:1404.3589v6 [cs.NI].

[6] Adam Dunkels,"ContikiMAC Radio Duty Cycling Protocol",(2011), T2011:13. ISSN 1100-3154.

[7] Mo Sha, Gregory Hackmann, Chenyang Lu, "Energy-Efficient Low Power Listening for Wireless Sensor Networks in Noisy Environments",(2013), IPSN'13, Philadelphia, Pennsylvania, USA.

[8] Krita Pattamasiriwat and Chaiporn Jaikaeo, "Evaluation of Low Power Listening MAC Protocol on Network Monitoring in Wireless Sensor Networks", (2017).

[9] Nader Mohamed, Jameela Al-Jaroodi, Imad Jawhar, and Sanja Lazarova-Molnar, "Failure Impact on Coverage in Linear Wireless Sensor Networks “,(2013), SPECTS.

[10] Rodrigue Domga Komguem, Razvan Stanica, Maurice Tchuente, Fabrice Valois, "Node Ranking in Wireless Sensor Networks with Linear Topology", (2017), IEEE Conference Publications.

[11] Zhengjie Wang, Xiaoguang Zhao and Xu Qian, "The Application and Issuse of Linear Wireless Sensor Networks", (2011), International Conference on System Science, Engineering Design and Manufacturing Informatization.

[12] Bing Wu, Jian Lin and Xiaoyan Xiong, "Design and Implementation of Intelligent Monitoring and Diagnosis System Based on WSN and MAS", (2011), WISM 2011.

[13] Pouria Zand, Arta Dilo and Paul Havinga, "A Distributed Network Management Scheme for Real-Time Monitoring and Process Control Applications in Wireless Industrial Automation",(2013), doi:10.3390/s130708239.

[14] Fahad Alduraibi, Noureddine Lasla, Mohamed Younis, "Coveragebased Node Placement Optimization in Wireless Sensor Network with Linear Topology", (2016), IEEE ICC.

[15] Zeenat Rehena, Rajesh Mukherjee, Sarbani Roy, Nandini Mukherjee, "Detection of Node Failure in Wireless Sensor Networks", (2014),AIMoC.

[16] L. B. Ruiz, J. M. Nogueira, and A. A. F. Loureiro, "a Management Architecture for wireless sensor networks",(2013), IEEE Communication Magazine.

[17] Amir Darehshoorzadeh and Azzedine Boukerche, "Underwater Sensor Networks: A New Challenge for Opportunistic Routing Protocols", (2015), IEEE Communications Magazine.

N. Ramanathan and et.al. "Sympathy for the Sensor Network Debugger," (2005), 3rd Embedded networked sensor systems, San Diego, USA.

[18] C. Hsin and M. Liu, "A Two-Phase Self-Monitoring Mechanism for Wireless Sensor Networks",(2006) Journal of Computer Communications special issue on Sensor Networks.

[19] Mounir Arioua ,Younes el Assari, Imad Ez-zazi, Ahmed el Oualkadi, "Multi-hop cluster based routing approach for wireless sensor". (2016), The 7th International Conference on Ambient Systems, Networks and Technologies.

\footnotetext{
*Corresponding Author
} 
AUTHOR's PROFILE

SHAHZAD ASHRAF received bachelor degree (B.E) in Computer Systems Engineering from Mehran University of Engineering \& Technology, Jamshoro Pakistan in 2004 and the Master degree (M.E) in Communication System and Networks in 2014 from Mehran University of Engineering \& Technology, Pakistan. Currently he is a Ph.D student in

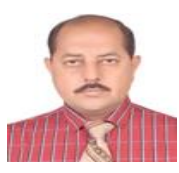
Information and Communication Engineering from College of Internet of
Things Engineering at Hohai University, Changzhou China. He has been an Assistant Professor at NFC Institute of Engineering and Technology, Multan Pakistan. His research interest include Underwater wireless sensor network, Computer hardware, Web Engineering and Graphics application.

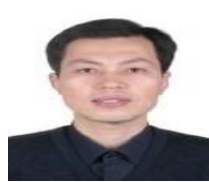

MINGSHENG GAO received the Ph.D. degree in radio engineering from Southeast University, Nanjing, China, in 2005. He is currently an associate professor with the Department of Information and Communication Engineering, Hohai University, China. Prior to that, he was a R\&D Engineer at Zhongxing Telecommunications, Ltd., Shenzhen, China from 1998 to 2005, and serving as a Research Staff in National University of singapore, Nanyang Technological University respectively, during 2007-2013. His current research interests include wireless sensor networks, underwater communications and smart grid.

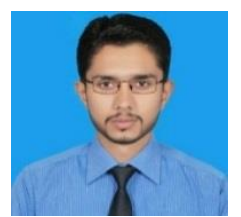

SYED KAMRAN HAIDER received B.S Telecommunication Engineering from Foundation university, Rawalpindi Pakistan in 2015. At present he is pursuing his Masters Degree from College of Internet of Things Engineering at Hohai University, Changzhou, China. His area of interest are Signal processing and wireless sensor networks.

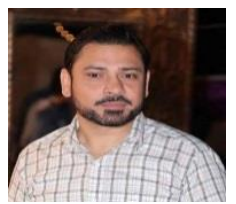

ZEESHAN RAZA received B.S degree in Computer Engineering from Sir Syed University of Engineering \& Technology Karachi Pakistan in 1999 while Masters degree in Electrical Engineering from University of Lahore, Pakistan in 2012. Currently he is a Ph.D student at University of Lahore, Pakistan in Electrical Engineering. His area of interest is Massive MIMO Multimedia Network and computer vision.

\footnotetext{
*Corresponding Author
} 